\title{
JAK2/STAT3 pathway as a therapeutic target in ovarian cancers
}

\author{
TOMOYUKI YOSHIKAWA ${ }^{1,2}$, MORIKAZU MIYAMOTO ${ }^{3}$, TADASHI AOYAMA ${ }^{3}$, \\ HIROAKI SOYAMA ${ }^{3}$, TOMOKO GOTO $^{3}$, JUNKO HIRATA ${ }^{3}$, AYAKO SUZUKI ${ }^{3}$, \\ ISAO NAGAOKA ${ }^{2}$, HITOSHI TSUDA ${ }^{4}$, KENICHI FURUYA $^{3}$ and MASASHI TAKANO ${ }^{1}$ \\ ${ }^{1}$ Department of Clinical Oncology, National Defense Medical College Hospital, Tokorozawa, Saitama 359-8513; \\ ${ }^{2}$ Department of Host Defense and Biochemical Research, Juntendo University, Graduate School of Medicine, \\ Tokyo 113-8431; Departments of ${ }^{3}$ Obstetrics and Gynecology and ${ }^{4}$ Basic Pathology, \\ National Defense Medical College Hospital, Tokorozawa, Saitama 359-8513, Japan
}

Received June 8, 2017; Accepted January 16, 2018

DOI: $10.3892 / \mathrm{ol} .2018 .8028$

\begin{abstract}
The activation of JAK2/STAT3 pathway has been reported to have critical roles in several solid tumors. The present study aimed to evaluate the correlation between JAK2/STAT3 activation and clinicopathological parameters in ovarian cancer types. Tissue microarrays made from the patients treated at the National Defense Medical College Hospital between 1984 and 2008 were evaluated using immunohistochemical (IHC) stainings. Medical charts of these patients including IHC results were retrospectively analyzed, and prognostic factors for progression-free survival and overall survival were evaluated. Among 341 enrolled patients, positive expression of p-STAT3 was observed in 95 cases (28\%). Positive p-STAT3 was an independent worse prognostic factor for overall survival in all the cases. Additionally, p-STAT3 expression was related with overall survival in patients with clear-cell histology, but not in serous histology. The effect of an inhibitor of STAT3, niclosamide, was evaluated in ovarian clear-cell cancer cells, and niclosamide treatment decreased expression of p-STAT3, leading to increased apoptosis in a dose-dependent manner in vitro. The activation of JAK2/STAT3 pathway had significant impact on survival of ovarian cancers, especially for the cases with clear-cell histology. Although further analyses are needed, suppression of this pathway could be a candidate for the treatment of ovarian cancers.
\end{abstract}

\section{Introduction}

Epithelial ovarian cancer, accounting for $>90 \%$ of primary ovarian neoplasms, had four major histological subtypes:

Correspondence to: Dr Masashi Takano, Department of Clinical Oncology, National Defense Medical College Hospital, 3-2 Namiki, Tokorozawa, Saitama 359-8513, Japan

E-mail: mastkn@ndmc.ac.jp

Key words: ovarian cancer, clear-cell, serous, JAK2/STAT3, STAT3 inhibitor, niclosamide
Serous, endometrioid, mucinous and clear-cell subtypes (1). Several reports evaluating biological and clinical behavior of ovarian cancers suggested that there have been several classifications in addition to histological subtypes (2-7).

The activation of JAK2/STAT3 pathway has been reported to play critical roles in several oncogenic processes including proliferation, survival, differentiation and angiogenesis in several solid tumors $(8,9)$. In ovarian cancers, some reports suggested have reported that the activation of STAT3 was associated with prognoses by univariate analysis $(10,11)$. In serous histological subtype, a report revealed that STAT3 polymorphisms were associated with unfavorable responses against platinum-based chemotherapy (12). In clear-cell subtype, it has been reported that IL-6R, an activator of JAK2/STAT3 signaling, was correlated with unfavorable survival by multivariate analysis, however, no relationship between prognoses and activation of phosphorylated STAT3 (p-STAT3) was observed (13).

Niclosamide (C13H8Cl2N2O4) is a small-molecule drug of the teniacide anthelmintic family that is effective against human tapeworms $(14,15)$. This agent was previously reported to have therapeutic activities in several cancers by inhibition of STAT3 $(14,16,17)$. However, there have been no reports evaluating whether inhibiting STAT3 signaling using this drug could modify the sensitivity to platinums in ovarian cancers.

The present study was conducted to evaluate the correlation between activation of JAK2/STAT3 pathway and clinicopathological parameters in ovarian cancers. Additionally, antitumor effect by inhibition of JAK2/STAT3 pathway was evaluated in vitro evaluation.

\section{Patients and methods}

Patients and tissue microarray. Tissue blocks from a total of 341 patients with epithelial ovarian carcinoma who received primal surgery at the National Defense Medical College Hospital (Tokorozawa, Japan) between 1984 and 2008 were used for the present study. A total of 341 patients who met the inclusion criteria were enrolled in this investigation: i) patients who received no prior chemotherapy before surgical therapy; ii) patients who were diagnosed to have epithelial 
ovarian cancers by pathological evaluation; iii) patients whose histological subtype was serous, endometrioid, mucinous, and clear-cell type; iv) patients whose medical information, and tissue blocks were available. The institutional ethical review board of National Defense Medical College approved the protocol of the present retrospective analyses. Comprehensive informed consent using tumor samples had been obtained from each patient at the time of primary treatment. After IRB approval, the notice of the protocol including the use patients' samples were open to the public, without any objection or rejection. So, all the samples of the patients were used in the present study.

Two core specimens, $1.5 \mathrm{~mm}$ in diameter, for each case were taken from cancer blocks and transferred to recipient blocks using a Tissue Microarrayer (Beecher Instrument, Silver Spring, MD, USA). All specimens were cut into 4- $\mu$ m-thick slices to make sections for immunohistochemical (IHC) staining. Satisfactory IHC staining was obtained in all cases.

Characteristics of the patients were shown in Table I. Median age of all patients was 53 years (range, $16-82$ years), and median follow-up duration was 58 months (range, 1-257 months). The number of patients according to the International Federation of Obstetrics and Gynecology (FIGO) classification was as follows: 126 in stage I, 40 in stage II, 129 in stage III, and 46 in stage IV, respectively. The subjects consisted of 197 patients with no residual tumors (RT) after a primary cytoreductive surgery, 47 with optimal surgery, and 97 with suboptimal surgery. A total of 90 patients were treated with taxanes and platinum chemotherapy: 78 cases with paclitaxel and carboplatin (TC) and 12 cases with docetaxel and carboplatin (DC) regimen. Platinum-based chemotherapy was used in 223 cases: 165 cases by combination with cyclophosphamide, adriamycin and cisplatin (CAP), 31 cases by chemotherapy with irinotecan and cisplatin (CPT-P), 15 cases by chemotherapy with etoposide and cisplatin (EP), and 12 cases by the other regimens.

Reagents/antibodies. Niclosamide was purchased from Selleck Chemicals (Houston, TX, USA). The primary antibodies against p-STAT3, XIAP, cleaved PARP, and $\beta$-actin and the secondary antibodies were obtained from Cell Signaling Technology (Danvers, MA, USA). The primary antibodies against STAT3 and p-JAK2 was obtained from Abcam (Cambridge, MA, USA).

IHC staining. For IHC staining, antibodies used were phospho-JAK2 (Y1007 + Y1008; rabbit monoclonal, 1:100; Abcam) and phospho-STAT3 (Tyr705; rabbit monoclonal, 1:50; Cell Signaling Technology, Inc.). Tissue microarray slides were deparaffinised in xylene and hydrated with ethanol. The slides for $\mathrm{p}-\mathrm{JAK} 2$ were boiled in an autoclave at $121^{\circ} \mathrm{C}$ for $15 \mathrm{~min}$ in citrate buffer $(10 \mathrm{mM}$, pH 6.0), and then allowed to cool at room temperature. The slides for p-STAT3 were pretreated in an electric pot at $98^{\circ} \mathrm{C}$ for $40 \mathrm{~min}$ in EDTA buffer ( $1 \mathrm{mM}, \mathrm{pH} 8.0)$. Endogenous peroxidase activity was blocked by $0.3 \% \mathrm{H}_{2} \mathrm{O}_{2}$ /methanol. The slides were incubated at $4{ }^{\circ} \mathrm{C}$ overnight with primary antibodies and reacted with the DAKO EnVision + system-horseradish peroxidase (HRP)-labelled polymer (DAKO Denmark A/S, Glostrup, Denmark) as a secondary antibody for $30 \mathrm{~min}$ at room temperature. Specific antigen-antibody reactions were visualized with $0.2 \%$ diaminobenzine tetrahydrochloride and hydrogen peroxide, and counterstained with Mayer haematoxylin. Positive staining was defined as the present of staining of $>10 \%$ of the nuclei.

Cell lines and culture conditions. Ovarian clear cell cancer cell line, KK, were used (18). These cell lines were grown as monolayer cultures in RPMI-1640 + Glutmax ${ }^{\mathrm{TM}}-\mathrm{I}$ (Invitrogen Japan KK, Tokyo, Japan) medium supplemented with $10 \%$ fetal bovine serum (Invitrogen Japan KK), $100 \mathrm{U}$ penicillin per $\mathrm{ml}$, and $100 \mathrm{mg}$ streptomycin per $\mathrm{ml}$ (Invitrogen Japan KK) in a humidified atmosphere of $5 \% \mathrm{CO}_{2}$ at $37^{\circ} \mathrm{C}$, and routinely tested for mycoplasma infection. Protein concentrations were determined by Bradford assay (Bio-Rad Laboratories, Hercules, CA, USA). KK cells were positive for expression of phospho-STAT3 in normal condition.

Cell proliferation and cytotoxicity assay. KK cells were seeded onto 96 -well plates at $\sim 1 \times 10^{4}$ or $4 \times 10^{4}$ cells $\mathrm{cm}^{-2}$ for cytotoxicity assays. Cell viability was determined by MTT method using Tetra Color ONE (Seikagaku Corporation, Tokyo, Japan) according to the manufacturer's instructions. Cell cytotoxicity by niclosamide was measured after 5 days from the treatment at different concentration. For the evaluation of protein expression, KK cells were harvested after $24 \mathrm{~h}$ from the treatment with niclosamide. The experiments were repeated three times, and mean values were used to construct the kinetic curves.

Preparation of cell lysate for western blot analysis. Protein lysates were extracted in RIPA buffer according to the manufacturer's instructions (Wako Pure Chemical Industries, Ltd., Osaka, Japan). Ten $\mu \mathrm{g}$ cytosolic fractions were and loaded onto Mini-PROTEIN TGXTM gel (Bio-Rad Laboratories). After electrophoresis, proteins were transferred to PVDF membranes using Trans-Blot ${ }^{\circledR}$ TurboTM Transfer System Transfer Pack (Bio-Rad Laboratories). Subsequently, the membranes were blocked for $1 \mathrm{~h}$ in $4 \%$ BSA in TBS with $0.5 \%$ Tween-20 (PBS-T) and incubated overnight at $4^{\circ} \mathrm{C}$ in primary antibody in TBS-T with $4 \%$ BSA. The following antibodies and concentrations were used: 1/2,500 rabbit STAT3 (Abcam), 1/2,500 rabbit p-STAT3, 1/2,500 rabbit anti-XIAP, 1/2,500 rabbit cleaved PARP, and 1/5,000 rabbit $\beta$-actin (all Cell Signaling Technology). After three washes with TBS-T, membranes were incubated for $1 \mathrm{~h}$ at room temperature using HRP-conjugated anti-rabbit secondary antibody as appropriate. After three washes with TBS-T, they were visualized using the ECL detection system (GE Healthcare UK Ltd., Chalfont, UK) by a LAS-3000 (Fujifilm, Tokyo, Japan). Protein expression was determined densitometrically and normalized against $\beta$-actin expression using Multi Gauge version 3.1 (Fujifilm).

Statistical analysis. Statistical analyses were performed using JMP Pro, version 11 (SAS Institution Inc., Cary, NC, USA). Progression-free survival (PFS) was defined as the interval between initial surgery and the date of disease progression. Overall survival (OS) was defined as the interval between initial surgery and death. The serum tumor markers including CA125 were not used for the definition of disease progression. 
Table I. Characteristics of the patients.

\begin{tabular}{|c|c|c|c|c|c|}
\hline \multirow[b]{2}{*}{ Variables } & \multicolumn{4}{|c|}{ Histology } & \multirow[b]{2}{*}{$\begin{array}{c}\text { Total } \\
\mathrm{n}=341\end{array}$} \\
\hline & $\begin{array}{l}\text { Serous } \\
n=144\end{array}$ & $\begin{array}{c}\text { Clear-cell } \\
n=85\end{array}$ & $\begin{array}{c}\text { Endometrioid } \\
n=52\end{array}$ & $\begin{array}{c}\text { Mucinous } \\
n=60\end{array}$ & \\
\hline \multicolumn{6}{|l|}{ Age (years) } \\
\hline $16-52$ & 59 & 43 & 25 & 32 & 159 \\
\hline $53-82$ & 85 & 42 & 27 & 28 & 182 \\
\hline \multicolumn{6}{|l|}{ FIGO stage } \\
\hline I & 17 & 44 & 24 & 41 & 126 \\
\hline II & 12 & 8 & 15 & 5 & 40 \\
\hline III & 79 & 29 & 10 & 11 & 129 \\
\hline IV & 36 & 4 & 3 & 3 & 46 \\
\hline \multicolumn{6}{|l|}{ Primary surgery } \\
\hline Complete surgery $(\mathrm{RT}=0 \mathrm{~cm})$ & 64 & 61 & 26 & 46 & 197 \\
\hline Optimal surgery $(\mathrm{RT} \leq 1 \mathrm{~cm})$ & 21 & 10 & 12 & 4 & 47 \\
\hline Suboptimal surgery $(\mathrm{RT}>1 \mathrm{~cm})$ & 59 & 14 & 14 & 10 & 97 \\
\hline \multicolumn{6}{|l|}{ Primary chemotherapy } \\
\hline Taxane + platinum & 64 & 11 & 12 & 3 & 90 \\
\hline Platinum-based therapy & 78 & 68 & 31 & 46 & 223 \\
\hline None & 2 & 6 & 9 & 11 & 28 \\
\hline
\end{tabular}

FIGO, The International Federation of Gynecology and Obstetrics; RT, residual tumor.

Table II. Expressions of p-JAK2 and p-STAT3 according to histological subtypes.

\begin{tabular}{|c|c|c|c|c|c|}
\hline \multirow[b]{2}{*}{ p-JAK2/p-STAT3 } & \multicolumn{4}{|c|}{ Histology } & \multirow[b]{2}{*}{$\begin{array}{c}\text { Total } \\
\mathrm{n}=341\end{array}$} \\
\hline & $\begin{array}{l}\text { Serous } \\
n=144\end{array}$ & $\begin{array}{c}\text { Clear-cell } \\
n=85\end{array}$ & $\begin{array}{l}\text { Endometrioid } \\
n=52\end{array}$ & $\begin{array}{c}\text { Mucinous } \\
n=60\end{array}$ & \\
\hline Positive/positive & 34 & 27 & 16 & 9 & 86 \\
\hline Positive/negative & 93 & 36 & 26 & 43 & 198 \\
\hline Negative/positive & 3 & 2 & 1 & 3 & 9 \\
\hline Negative/negative & 14 & 20 & 9 & 5 & 48 \\
\hline P-value ${ }^{a}$ & 0.560 & 0.004 & 0.138 & 0.191 & 0.034 \\
\hline
\end{tabular}

${ }^{\text {a}}$ Fisher's exact probability. p-JAK2, phosphorylated-Janus kinase 2; STAT3, signal transducer and activator of transcription 3.

Comparisons were evaluated with the chi-square test or the Fisher's exact probability test when appropriate. PFS and OS curves were generated using the method of Kaplan-Meier. The comparison of the survival distributions was made using a log-rank test. Cox's proportional hazards model was used for univariate and multivariate analysis. $\mathrm{P}<0.05$ was considered to indicate a statistically significant difference.

\section{Results}

A total of 341 patients with epithelial ovarian cancers were identified. Among them, positive nuclear p-STAT3 expression was observed in 95 cases $(28 \%)$, positive nuclear p-JAK2 expression was in 284 (83\%). Expression pattern of p-STAT3 and p-JAK2 according to histological subtypes were shown in Table II. There was a significant association of p-JAK2 expression and $\mathrm{p}-\mathrm{STAT} 3$ in all samples $(\mathrm{P}=0.034)$. Significant association of these protein expressions was only observed in clear-cell histology $(\mathrm{P}=0.004)$. There were no significant differences in age, FIGO stage, RT size at primary surgery, histological subtype and primary chemotherapy according to p-STAT3 expression (data not shown).

The patients with negative p-STAT3 had slightly better PFS compared with the cases with positive p-STAT3, although the difference was not statistically significant $(\mathrm{P}=0.055$; hazard ratio, 1.37; 95\% confidence interval, 0.98-1.90; Fig. 1A). The 

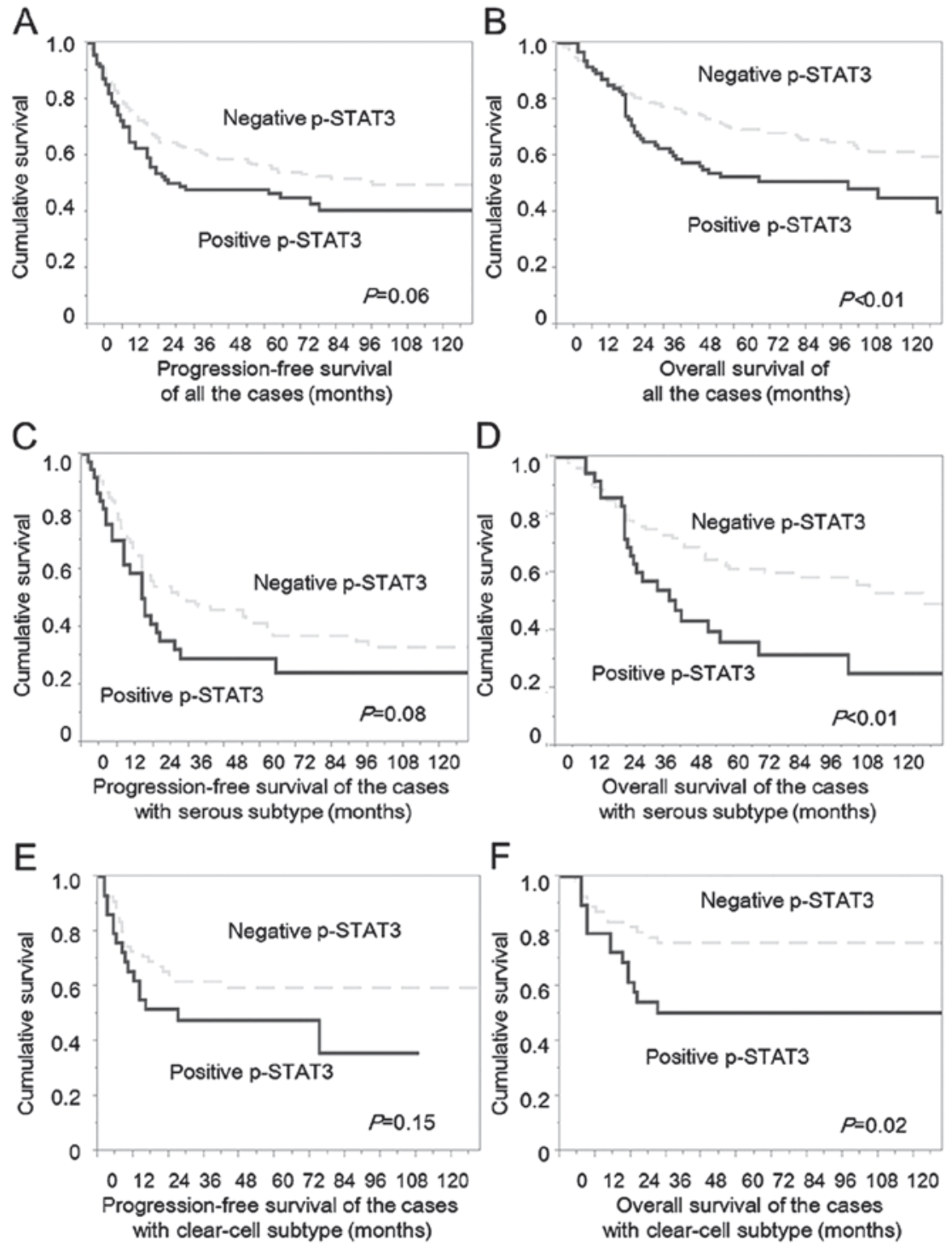

Figure 1. PFS and OS curves of the cases with ovarian cancers according to p-STAT3 expression. (A) PFS of all the patients. The patients with negative p-STAT3 had a slightly better progression-free survival compared with the cases with positive p-STAT3, although the difference was not statistically significant $(\mathrm{P}=0.055$; hazard ratio, $1.37 ; 95 \%$ confidence interval, $0.98-1.90)$. (B) OS of all the patients. The patients with negative p-STAT3 had significantly better OS compared with the cases with positive p-STAT3 ( $\mathrm{P}=0.002$; hazard ratio, 1.75; $95 \%$ confidence interval, 1.22-2.48). (C) PFS of the patient with serous subtype. The patients with negative p-STAT3 had slightly better progression-free survival compared with the cases with positive p-STAT3, although the difference was not statistically significant ( $\mathrm{P}=0.077$; hazard ratio, 1.49; $95 \%$ confidence interval, $0.93-2.31)$. (D) OS of the patient with serous subtype. The patients with negative p-STAT3 had significantly better OS compared with the cases with positive p-STAT3 (P=0.002; hazard ratio, 2.10; 95\% confidence interval, 1.27-3.42). (E) PFS of the patient with clear-cell subtype. The patients with negative p-STAT3 had slightly better progression-free survival compared with the cases with positive $\mathrm{p}$-STAT3, although the difference was not statistically significant ( $\mathrm{P}=0.147$; hazard ratio, 1.59 ; $95 \%$ confidence interval, $0.83-3.03$ ). (F) OS of the patient with clear-cell subtype. The patients with negative p-STAT3 had significantly better OS compared with the cases with positive p-STAT3, although the difference was not statistically significant $(\mathrm{P}=0.019$; hazard ratio, 2.32; 95\% confidence interval, 1.11-4.89). Dotted line, cases with negative p-STAT3; solid line, cases with positive p-STAT3. PFS, progression-free survival; OS, overall survival; p-STAT3, phosphorylated STAT3.

patients with negative p-STAT3 had significantly improved OS compared with those with positive p-STAT3 $(\mathrm{P}=0.002$; hazard ratio, 1.75; 95\% confidence interval, 1.22-2.48; Fig. 1B). In contrast, positive p-JAK2 did not influence OS $(\mathrm{P}=0.142$; hazard ratio, $1.47 ; 95 \%$ confidence interval, $0.90-2.59)$ and PFS $(\mathrm{P}=0.414$, hazard ratio, 1.20; 95\% confidence interval, $0.79-1.89 ; \mathrm{P}=0.409)$ in the analyses in all the patients. Multivariate analyses for OS revealed that positive expression of p-STAT3 was identified as an independent prognostic factor (hazard ratio, 1.48; 95\% confidence interval, 1.02-2.11; $\mathrm{P}=0.039$, Table III), in addition to stage, histology, and RT diameter.
In the analyses of serous type, positive p-STAT3 was observed in 37 cases $(26 \%)$, and positive nuclear p-JAK2 expression was detected in 127 cases (88\%). Primary surgery was suboptimal in $21(57 \%)$ of 37 positive p-STAT3 and in 38 (36\%) of 107 negative p-STAT3. Positive expression of p-STAT3 was significantly associated with larger RT size ( $\mathrm{P}=0.024$, complete+optimal vs. suboptimal). There were no significant differences in age, FIGO stage, chemotherapy and positive p-JAK2 according to positive p-STAT3. The patients with negative p-STAT3 had slightly better PFS compared with the cases with positive p-STAT3, although the difference was not statistically significant $(\mathrm{P}=0.077$; hazard ratio, 1.49 ; 


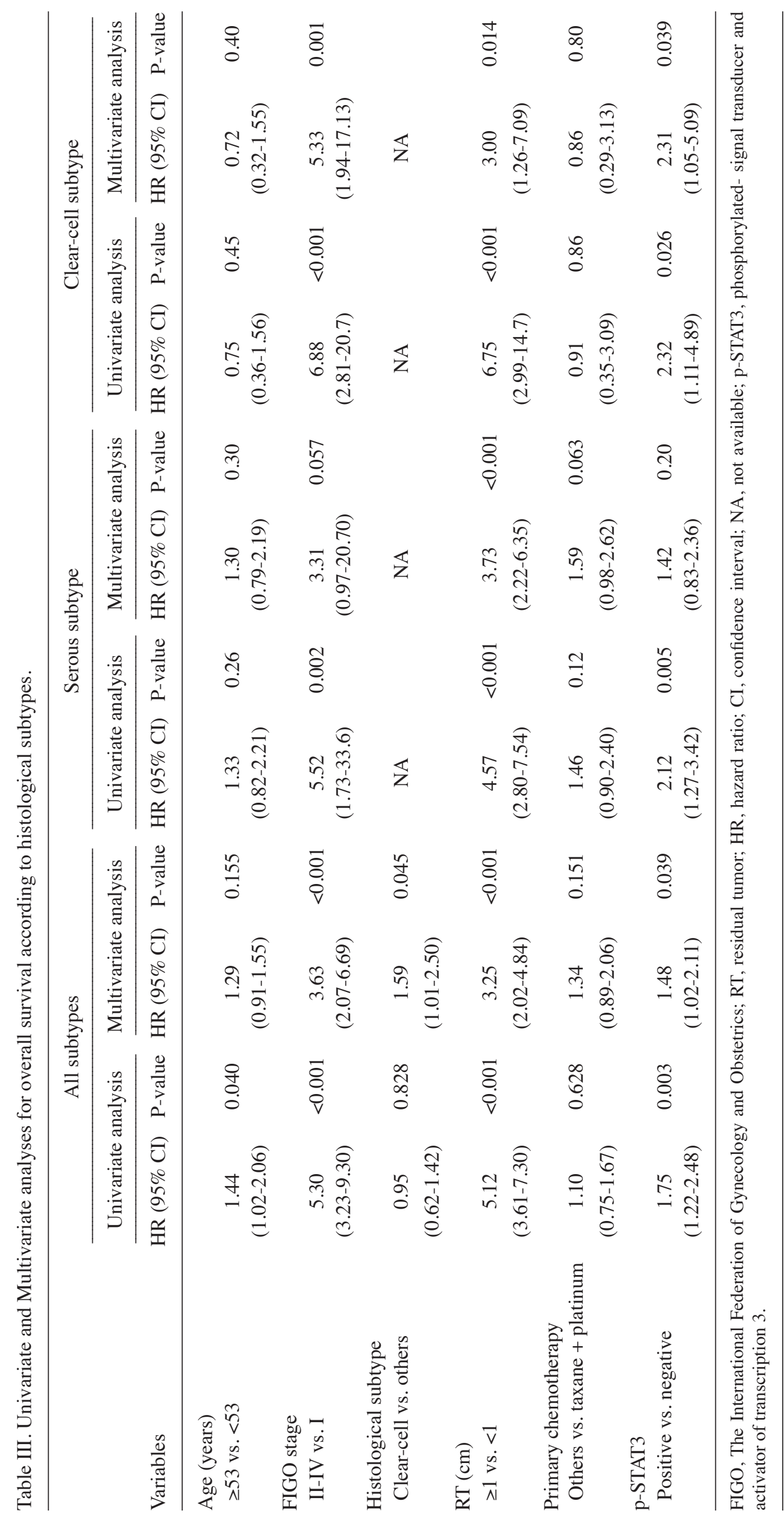




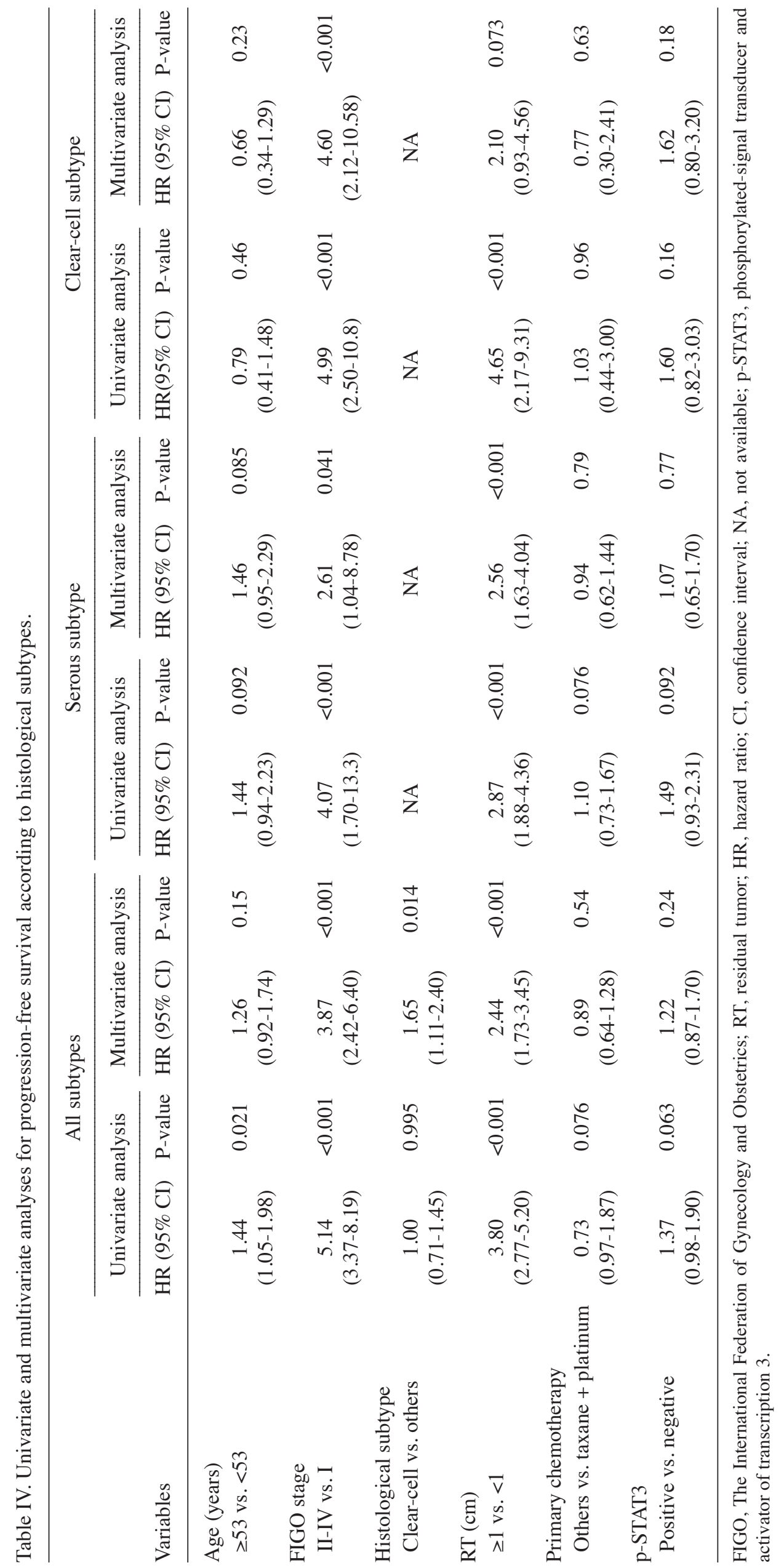




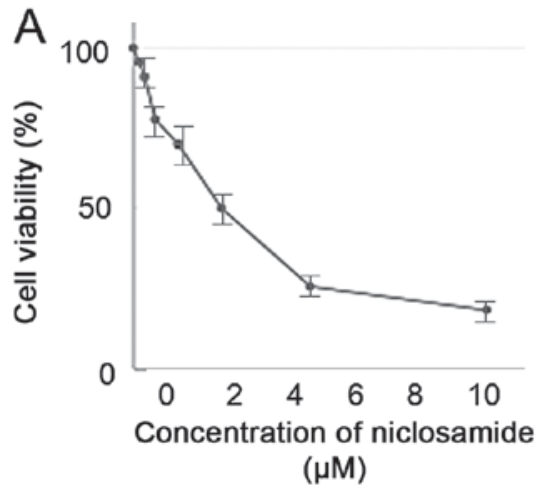

$(\mu \mathrm{M})$

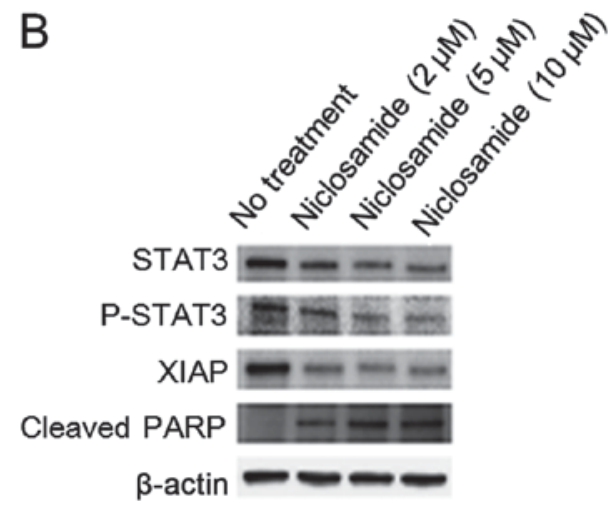

Figure 2. STAT 3 inhibitor, niclosamide, had the antitumor activity for ovarian clear cell cancer cell lines, KK. (A) Niclosamide had antitumor effect for ovarian clear-cell carcinoma cell line, KK. (B) Western blot analysis revealed the downregulation of p-STAT3, and XIAP proteins and increased expression of cleaved-PARP by treatment with niclosamide in a dose-dependent manner. Cell viability was assessed using MTT assay after $24 \mathrm{~h}$ from the treatment with niclosamide. Equivalent amounts $(10 \mu \mathrm{g})$ of protein were subjected to SDS-PAGE and blotted with anti-STAT3, anti-phospho-STAT3, anti-XIAP, anti-cleaved-PARP, and anti- $\beta$-actin antibodies.

95\% confidence interval, 0.93-2.31; Fig. 1C). Additionally, the patients with negative p-STAT3 had significantly better OS compared with the cases with positive p-STAT3 $(\mathrm{P}=0.002$; hazard ratio, 2.10; 95\% confidence interval, 1.27-3.42 Fig. 1D). In multivariate analyses, positive p-STAT3 was not selected as an independent prognostic factor for both OS and PFS in the analyses of the patients with serous histology (Tables III and IV).

In the analyses of a total of 85 patients with clear-cell histology, positive nuclear p-STAT3 was observed in 29 cases (34\%), and positive nuclear p-JAK2 expression was in observed in 63 cases (74\%). Positivity of p-STAT3 was significantly related with positivity of p-JAK2 ( $\mathrm{P}=0.004)$ (Table II). There were no significant differences in age, FIGO stage and RT size according to p-STAT3 expression. The patients with negative p-STAT3 had slightly better PFS compared with the cases with positive p-STAT3, although the difference was not statistically significant $(\mathrm{P}=0.147$; hazard ratio, 1.59 ; $95 \%$ confidence interval, 0.83-3.03) (Fig. 1E). Further, the patients with negative p-STAT3 had significantly better OS compared with the cases with positive p-STAT3 ( $\mathrm{P}=0.019$; hazard ratio, 2.32; 95\% confidence interval, 1.11-4.89; Fig. 1F). Multivariate analyses for OS revealed that positive expression of p-STAT3 was identified as an independent prognostic factor (hazard ratio, 2.31; 95\% confidence interval, 1.05-5.09; $\mathrm{P}=0.039$ ), in addition to stage, histology (Table III).

Cell viability of KK cells decreased by the treatment with niclosamide in a dose-dependent manner. Experiments were repeated three times with similar results. Additionally, niclosamide treatment decreased expression of p-STAT3, and inhibitor of apoptosis protein XIAP, and increased apoptosis related protein cleaved PARP in a dose-dependent manner in KK cells (Fig. 2).

\section{Discussion}

In the present study, activation of STAT3 was identified as an independent prognostic factor for ovarian cancers, especially in the patients with clear cell histology. To our knowledge, it is the first report to demonstrate the correlation between the activation of STAT3 and unfavorable survival for
OS by multivariate analysis in ovarian cancers including four major histological subtypes. Hazard ratio by p-STAT3 status for OS in multivariate analyses in all cases, clear-cell type and serous type was $1.48,2.31$ and 1.42 . These results lead us to conclude that the activation of STAT3 plays critical roles in survival in ovarian cancers with clear-cell type in particular. Positive rate of p-STAT3 in all cases, clear-cell subtype, and serous type was 28,34 and $26 \%$ in the present study. Previous reports documented that positive rate of p-STAT3 expression ranged from 29 to $58 \%$ of all histological subtypes, and from 39 to $90 \%$ in clear-cell subtype $(10,11,13,19)$. Although the frequency of positive p-STAT3 in the present study was a slightly lower than those of the published findings, the criteria of the present study clearly enabled us to distinguish the patients with worse OS from all ovarian cancer patients, and especially from the patients with clear cell histology.

Previous reports demonstrated that positive nuclear p-STAT3 expression was associated with histological grade, lymph node metastasis, clear-cell type, serous type and stage $(10,11)$. In the present study, positive p-STAT3 was associated with RT size after primary surgery in serous type. To the best of our knowledge, it is the first report to demonstrate the association between the activation of STAT3 and RT status after primary surgery in ovarian cancers. In the present study, the low affinity between positive p-STAT3 and poor outcome in serous type by multivariate analysis is probably attributable to the relevance that depends strongly on RT size.

It was demonstrated that niclosamide had therapeutic activities via apoptosis in cancer cell line established from ascites of a patient with ovarian clear cell carcinoma showing less sensitivity to cisplatin (18). Additionally, oral niclosamide inhibited tumor growth and progression in an intraperitoneal xenograft mouse model representative of human ovarian cancer without significant side effects (20). Recently, niclosamide has been shown to inhibit several pathways including NF-кB, Notch, ROS, mTORC 1 , and Wnt/ $\beta$-catenin pathway $(16,21-25)$. So there might be several mechanisms of anti-neoplastic activity in niclosamide other than inhibition of JAK2/STAT3 pathway.

Several studies evaluated inhibition of JAK2/STAT3 pathway in ovarian cancers, and it is suggested that activation of STAT3 was associated with proliferation, chemo-resistance, 
and cancer stem-cell phenotype (26-30). So, it was speculated that worse PFS in the patients with positive p-STAT3 tumors was contributed by a high abundance of the patients that showed chemo-resistance of primary tumors, although significance was not obtained $(\mathrm{P}=0.055)$. Additionally, significantly worse $\mathrm{OS}$ in the patients with positive p-STAT3 tumors might be attributed by acquired chemo-resistance caused by stem-cell phenotype in recurrent tumors. Also, a study demonstrated that inhibition of STAT3 activation by JAK2-specific inhibitor AG490 blocked STAT3 phosphorylation, cell motility, and induction IL-6 production (31). So, IL-6 pathway might have contributed to worse prognoses of clear-cell ovarian cancers. Finally, a kinase inhibitor of JAK1 and JAK2, Ruxolitinib Phosphate, is now evaluated in phase $1 / 2$ study for Mullerian cancers in combination with paclitaxel and carboplatin (32). Nevertheless, it is speculated that niclosamide would show activity against ovarian cancer patients whose tumors had JAK2/STAT3 activation.

The activation of JAK2/STAT3 pathway had significant impact on survival of ovarian cancers, especially for the cases with clear-cell histology. Although further analyses are needed, suppression of this pathway could be a candidate for the treatment of ovarian cancers, especially chemotherapy-resistant clear cell type.

\section{Acknowledgements}

The authors would like to thank Ms. Hiromi Kubota for their contribution to this study.

\section{References}

1. Jelovac D and Armstrong DK: Recent progress in the diagnosis and treatment of ovarian cancer. CA Cancer J Clin 61: 183-203, 2011.

2. Pignata S, Cannella L, Leopardo D, Pisano C, Bruni GS and Facchini G: Chemotherapy in epithelial ovarian cancer. Cancer Lett 303: 73-83, 2011.

3. Tothill RW, Tinker AV, George J, Brown R, Fox SB, Lade S, Johnson DS, Trivett MK, Etemadmoghadam D, Locandro B, et al: Novel molecular subtypes of serous and endometrioid ovarian cancer linked to clinical outcome. Clin Cancer Res 14: 5198-5208, 2008.

4. Bast RC Jr, Hennessy B and Mills GB: The biology of ovarian cancer: New opportunities for translation. Nat Rev Cancer 9: 415-428, 2009

5. Takano M, Kikuchi Y, Yaegashi N, Kuzuya K, Ueki M, Tsuda H, Suzuki M, Kigawa J, Takeuchi S, Tsuda H, et al: Clear cell carcinoma of the ovary: A retrospective multicentre experience of 254 patients with complete surgical staging. Br J Cancer 94 1369-1374, 2006.

6. Takano M, Sugiyama T, Yaegashi N, Suzuki M, Tsuda H, Sagae S, Udagawa Y, Kuzuya K, Kigawa J, Takeuchi S, et al: The impact of complete surgical staging upon survival in early-stage ovarian clear cell carcinoma: A multi-institutional retrospective study. Int J Gynecol Cancer 19: 1353-1357, 2009.

7. Takano M, Tsuda H and Sugiyama T: Clear cell carcinoma of the ovary: Is there a role of histology-specific treatment? J Exp Clin Cancer Res 31: 53, 2012.

8. Yu H and Jove R: The STATs of cancer-new molecular targets come of age. Nat Rev Cancer 4: 97-105, 2004.

9. Kong H, Zhang Q, Zeng Y, Wang H, Wu M, Zheng T, Zeng Y and Shi H: Prognostic significance of STAT3/phosphorylated-STAT3 in tumor: A meta-analysis of literatures. Int J Clin Exp Med 8: 8525-8539, 2015.

10. Rosen DG, Mercado-Uribe I, Yang G, Bast RC Jr, Amin HM, Lai R and Liu J: The role of constitutively active signal transducer and activator of transcription 3 in ovarian tumorigenesis and prognosis. Cancer 107: 2730-2740, 2006.
11. Min $\mathrm{H}$ and Wei-hong $\mathrm{Z}$ : Constitutive activation of signal transducer and activator of transcription 3 in epithelial ovarian carcinoma. J Obstet Gynaecol Res 35: 918-925, 2009.

12. Permuth-Wey J, Fulp WJ, Reid BM, Chen Z, Georgeades C, Cheng JQ, Magliocco A, Chen DT and Lancaster JM: STAT3 polymorphisms may predict an unfavorable response to first-line platinum-based therapy for women with advanced serous epithelial ovarian cancer. Int J Cancer 138: 612-619, 2016.

13. Yanaihara N, Hirata Y, Yamaguchi N, Noguchi Y, Saito M, Nagata C, Takakura S, Yamada K and Okamoto A: Antitumor effects of interleukin-6 (IL-6)/interleukin-6 receptor (IL-6R) signaling pathway inhibition in clear cell carcinoma of the ovary. Mol Carcinog 55: 832-841, 2016.

14. You S, Li R, Park D, Xie M, Sica GL, Cao Y, Xiao ZQ and Deng X: Disruption of STAT3 by niclosamide reverses radioresistance of human lung cancer. Mol Cancer Ther 13: 606-616, 2014.

15. Lateef M, Zargar SA, Khan AR, Nazir M and Shoukat A: Successful treatment of niclosamide- and praziquantel-resistant beef tapeworm infection with nitazoxanide. Int J Infect Dis 12: 80-82, 2008.

16. Ren X, Duan L, He Q, Zhang Z, Zhou Y, Wu D, Pan J, Pei D and Ding K: Identification of niclosamide as a new small-molecule inhibitor of the STAT3 signaling pathway. ACS Med Chem Lett 1: 454-459, 2010.

17. Xiang D, Yuan Y, Chen L, Liu X, Belani C and Cheng $\mathrm{H}$ : Niclosamide, an anti-helminthic molecule, downregulates the retroviral oncoprotein Tax and pro-survival Bcl-2 proteins in HTLV-1-transformed T lymphocytes. Biochem Biophys Res Commun 464: 221-228, 2015.

18. Sasa H, Ishii K, Hirata J, Kikuchi Y, Nagata I, Kawai T, Senoo A, Sugita M, Sugishita T and Tenjin Y: Establishment and characterization of a CA-125-producing human ovarian clear cell carcinoma cell line. Hum Cell 6: 279-286, 1993 (In Japanese).

19. Anglesio MS, George J, Kulbe H, Friedlander M, Rischin D, Lemech C, Power J, Coward J, Cowin PA, House CM, et al: IL6-STAT3-HIF signaling and therapeutic response to the angiogenesis inhibitor sunitinib in ovarian clear cell cancer. Clin Cancer Res 17: 2538-2548, 2011.

20. Arend RC, Londoño-Joshi AI, Samant RS, Li Y, Conner M, Hidalgo B, Alvarez RD, Landen CN, Straughn JM and Buchsbaum DJ: Inhibition of Wnt/ $\beta$-catenin pathway by niclosamide: A therapeutic target for ovarian cancer. Gynecol Oncol 134: 112-120, 2014

21. King ML, Lindberg ME, Stodden GR, Okuda H, Ebers SD, Johnson A, Montag A, Lengyel E, MacLean Ii JA and Hayashi K: WNT7A/ $\beta$-catenin signaling induces FGF1 and influences sensitivity to niclosamide in ovarian cancer. Oncogene 34: 3452-3462, 2015.

22. Jin Y, Lu Z, Ding K, Li J, Du X, Chen C, Sun X, Wu Y, Zhou J and Pan J: Antineoplastic mechanisms of niclosamide in acute myelogenous leukemia stem cells: Inactivation of the NF-kappaB pathway and generation of reactive oxygen species. Cancer Res 70: 2516-2527, 2010.

23. Chen M, Wang J, Lu J, Bond MC, Ren XR, Lyerly HK, Barak LS and Chen W: The anti-helminthic niclosamide inhibits Wnt/Frizzled1 signaling. Biochemistry 48: 10267-10274, 2009.

24. Sack U, Walther W, Scudiero D, Selby M, Kobelt D, Lemm M, Fichtner I, Schlag PM, Shoemaker RH and Stein U: Novel effect of antihelminthic Niclosamide on S100A4-mediated metastatic progression in colon cancer. J Natl Cancer Inst 103: 1018-1036, 2011.

25. Pan JX, Ding K and Wang CY: Niclosamide, an old antihelminthic agent, demonstrates antitumor activity by blocking multiple signaling pathways of cancer stem cells. Chin J Cancer 31: 178-184, 2012.

26. Fujiwara Y, Takaishi K, Nakao J, Ikeda T, Katabuchi H, Takeya M and Komohara Y: Corosolic acid enhances the antitumor effects of chemotherapy on epithelial ovarian cancer by inhibiting signal transducer and activator of transcription 3 signaling. Oncol Lett 6: 1619-1623, 2013.

27. Abubaker K, Luwor RB, Escalona R, McNally O, Quinn MA, Thompson EW, Findlay JK and Ahmed N: Targeted disruption of the JAK2/STAT3 pathway in combination with systemic administration of paclitaxel inhibits the priming of ovarian cancer stem cells leading to a reduced tumor burden. Front Oncol 4: 75, 2014. 
28. Abubaker K, Luwor RB, Zhu H, McNally O, Quinn MA, Burns CJ, Thompson EW, Findlay JK and Ahmed N: Inhibition of the JAK2/STAT3 pathway in ovarian cancer results in the loss of cancer stem cell-like characteristics and a reduced tumor burden. BMC Cancer 14: 317, 2014.

29. Gritsina G, Xiao F, O'Brien SW, Gabbasov R, Maglaty MA Xu RH, Thapa RJ, Zhou Y, Nicolas E, Litwin S, et al: Targeted blockade of JAK/STAT3 signaling inhibits ovarian carcinoma growth. Mol Cancer Ther 14: 1035-1047, 2015.

30. Zhong LX, Li H, Wu ML, Liu XY, Zhong MJ, Chen XY, Liu J and Zhang Y: Inhibition of STAT3 signaling as critical molecular event in resveratrol-suppressed ovarian cancer cells. J Ovarian Res 8: 25, 2015.
31. Colomiere M, Ward AC, Riley C, Trenerry MK, Cameron-Smith D, Findlay J, Ackland L and Ahmed N: Cross talk of signals between EGFR and IL-6R through JAK2/STAT3 mediate epithelial-mesenchymal transition in ovarian carcinomas. Br J Cancer 100: 134-144, 2009.

32. U.S. National Library of Medicine, National Cancer Institute (NCI): Ruxolitinib phosphate, paclitaxel, and carboplatin in treating patients with stage III-IV epithelial ovarian, fallopian tube, or primary peritoneal cancer. https://clinicaltrials. gov/ct2/show/NCT02713386. Accessed March 18, 2016. 